\title{
A relação entre 0 envelhecimento e a habilidade de escuta dicótica em indivíduos com mais de 50 anos
}

\author{
The relationship between aging and dichoticlistening ability in individuals aged \\ over 50 years

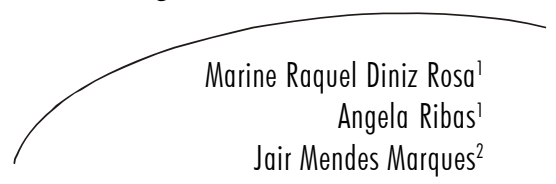

Resumo

Objetivo: estudar a habilidade de escuta dicótica de um grupo de indivíduos com mais de 50 anos e verificar as relações entre processamento auditivo, envelhecimento e perda auditiva. Método: foram avaliados 40 indivíduos na faixa etária de 50 a 83 anos, subdivididos em Grupo A (normo-ouvintes), grupo B (indivíduos com audição normal para idade) e grupo C (indivíduos com perda auditiva). Realizaram-se os seguintes procedimentos: anamnese, inspeção otológica, exames audiológicos e avaliação do processamento auditivo por meio da aplicação dos testes dicóticos de dissílabos alternados e dígitos. Resultados: verificou-se um transtorno do processamento auditivo em todos os grupos avaliados; o grau de alteração foi mais importante no grupo C; em todos os grupos, a habilidade auditiva mais afetada foi a de atenção seletiva. Conclusão: observou-se que o avanço da idade é um fator predisponente para alterações do processamento auditivo, em especial para a escuta dicótica, sendo que este pode, ainda, receber influência de perdas auditivas periféricas.

\section{Abstract}

Objective: To study the dichotic listening ability of a group of individuals over 50 years old and to verify the relationship between auditory processing, aging and hearing loss. Method: 40 individuals aged from 50 to 83 years were evaluated, subdivided into group A (normal listeners), group B (individuals with normal audition for their age) and group C (individuals with hearing loss). The following procedures were performed: anamnesis, otological

\footnotetext{
Universidade Tuiuti do Paraná. Curitiba, PR, Brasil

${ }^{1}$ Curso de Fonoaudiologia.

${ }^{2}$ Programa de Pós-graduação em Distúrbios da Comunicação

Correspondência / Correspondence

Ângela Ribas

E-mail: angela.ribas@utp.br
}

\author{
Palavras-chave: \\ Audiologia. \\ Audição. Envelhe- \\ cimento. Perda \\ Auditiva. Percepção \\ Auditiva.
}


inspection, audiological exams and evaluation of auditory processing by the administration of the dichotic tests of alternate disyllables and digits. Results: A disorder of auditory processing was verified in all the groups evaluated; the degree of alteration was more important in group C; in all groups the most affected auditory ability was selective attention. Conclusion: It was observed that the aging progress is a susceptible factor for alterations of auditory processing, especially for dichotic listening in view of the fact that this can still receive influences from peripheral auditory losses.
Key words:

Audiology. Hearing. Aging. Hearing Loss. Auditory Perception.
INTRODUÇÃO

Do ponto de vista demográfico, segundo Carvalho \& Andrade, ${ }^{1}$ envelhecer significa aumentar o número de anos vividos. Paralelamente à evolução cronológica, coexistem fenômenos de natureza biológica, psíquica e social importantes para a percepção da idade e do envelhecimento.

O crescimento da população de idosos, em números absolutos e relativos, é um fenômeno mundial e está ocorrendo em um nível sem precedentes. A população mundial está vivendo uma fase de declínio nos índices de natalidade e mortalidade, com consequente aumento da expectativa de vida. Estima-se que em 2020 existirão mais de um bilhão de pessoas com idade igual ou superior a 60 anos em todo o mundo. ${ }^{2}$

Tal assertiva aponta para o envelhecimento da população e, consequentemente, para o aumento das demandas relacionadas à saúde desta faixa etária. Segundo Papáleo Netto $\&$ Borgonovi, ${ }^{3} \mathrm{O}$ envelhecimento caracteriza-se por ser um processo dinâmico e progressivo, no qual as alterações, tanto morfológicas como funcionais e bioquími- cas, vão alterando progressivamente o organismo, tornando-o mais suscetível às agressões físicas que terminam por levá-lo à morte. A diminuição da capacidade funcional de órgãos e tecidos é a principal característica do envelhecimento e acarreta um risco aumentado de doenças crônico-degenerativas e o declínio do status do idoso na família e na sociedade. A gradual perda de energia física e produtividade econômica tende a isolá-lo e privá-lo de fontes de informação e comunicação.

Diversas ciências têm laços de afinidade com a geriatria e a gerontologia, por investigarem, de maneira interdisciplinar, as questões envolvidas com o envelhecimento humano. Uma delas, foco deste trabalho, é a Fonoaudiologia.

A Fonoaudiologia estuda os aspectos relacionados à comunicação humana, seus distúrbios e formas de tratamento, envolvendo a audição, o equilíbrio, a fala, a linguagem oral e escrita e a voz. Um dos aspectos da comunicação estudados pela Fonoaudiologia é a audição, tanto do ponto de vista periférico (estruturas do ouvido) quanto central (vias auditivas e córtex auditivo). 
Pesquisas demonstram que o ouvido humano atinge sua maturidade por volta dos 18-20 anos. A partir desta faixa etária, o órgão auditivo começa a envelhecer, seja por perda de células sensoriais, degeneração neurológica, exposição a agentes ototóxicos ou ruído. As vias auditivas centrais e o cérebro também sofrem alterações ao longo da vida, e problemas relacionados à atenção auditiva, memória, codificação e decodificação podem ser encontrados em populações idosas. ${ }^{4}$

Estes problemas, sejam de ordem periférica, como perdas auditivas, ${ }^{5}$ ou de ordem central, as chamadas desordens de processamento auditivo, ${ }^{6}$ quando afetam o idoso, acabam por desencadear problemas de comunicação importantes, que por sua vez geram dificuldades de adaptação social, ocupacional e familiar. É muito comum indivíduos idosos referirem que são capazes de ouvir, mas não entendem a fala.

No idoso, a presbiacusia é o tipo de alteração auditiva mais comum. Trata-se de uma perda auditiva bilateral para sons de altas frequências que pode gerar uma desordem do processamento auditivo por privação sensorial que afeta diretamente o mecanismo cerebral. Por processamento auditivo entendem-se as habilidades auditivas relativas à codificação, decodificação e interpretação dos estímulos em nível de sistema nervoso central, ${ }^{7}$ e a desordem do processamento auditivo refere-se exatamente às alterações ou dificuldades de processamento da informação auditiva. Nestes casos, a perda das informações acústicas diminui a probabilidade de se entender a fala e a interpretação dos sinais complexos. ${ }^{8}$

Uma das melhores maneiras de intervir na diminuição auditiva e na dificuldade em entender a fala é adaptar um aparelho de amplificação sonora individual, também conhecido por prótese auditiva. Entretanto, é comum encontrar, na prática clínica da Fonoaudiologia, mesmo após várias tentativas, pacientes idosos que não se adaptam a ele. Essa dificuldade na adaptação se deve, muitas vezes, a uma desordem do processamento auditivo, o que pode limitar o benefício do aparelho e influenciar na reabilitação de forma negativa. ${ }^{9}$

Tendo por bases estes pressupostos, o objetivo deste estudo foi analisar a qualidade das funções auditivas periféricas e centrais de um grupo de indivíduos com idade entre 50 e 83 anos, por meio de testes auditivos dicóticos, determinando suas peculiaridades quando isoladas as variáveis idade e perda auditiva periférica.

\section{MATERIAL E MÉTODO}

Esta pesquisa foi realizada no Laboratório de Audiologia da Clínica de Fonoaudiologia da Universidade Tuiuti do Paraná (UTP). A amostra foi composta por 40 indivíduos, com idade variando entre 50 e 83 anos, sendo 24 do gênero feminino e 16 do masculino, tendo em vista a dificuldade em encontrar indivíduos com idade acima de 
50 anos com audição dentro dos padrões de normalidade.

Todos os sujeitos da amostra foram submetidos a anamnese específica, avaliação audiológica básica (audiometria tonal limiar e imitanciometria) e exame de processamento auditivo (testes dicóticos SSW e Dígitos). Estes testes foram escolhidos por avaliarem os dois ouvidos ao mesmo tempo, com estímulos diferentes e competitivos entre si, o que permite verificar a capacidade que o indivíduo possui de realizar algumas tarefas auditivas complexas, como: atenção seletiva, fechamento auditivo, análise e síntese e organização de eventos sonoros no tempo.

Os exames foram realizados em cabine acústica, por meio do audiômetro Interacoustics, modelo AC-40, devidamente calibrado e acoplado a um CD player. Os resultados obtidos foram organizados em gráficos e tabelas e analisados através do progra- ma SPHINX LEXICA. Para análise dos resultados do exame de processamento auditivo e para determinar relação de melhor ouvido, utilizou-se o teste de WILCO$\mathrm{XON}$, adotando-se como valores significantes quando $\mathrm{p}<0,05$.

Este estudo é do tipo descritivo, tendo em vista seu objetivo primordial, a descrição das características de determinada população, fenômeno e suas relações. Foi aprovado pelo Comitê de Ética e Pesquisa da Universidade Tuiuti do Paraná-UTP (aprovação $n^{\circ} 074 / 2005$ ) e todos os participantes do estudo assinaram o termo de consentimento livre e esclarecido.

\section{RESULTADOS}

A avaliação audiológica básica permitiu qualificar a amostra e definir algumas condições auditivas dos indivíduos avaliados. Os resultados estão especificados na tabela 1.

Tabela 1 - Número de sujeitos de acordo com as características auditivas periféricas $(\mathrm{N}=40)$. Tuiutí, PR, 2007.

\begin{tabular}{llll}
\hline & Feminino & Masculino & Faixa Etária \\
\hline $\begin{array}{l}\text { Limiares auditivos dentro da } \\
\text { normalidade }\end{array}$ & $9(22,5 \%)$ & $1(2,5 \%)$ & 50 a 68 anos \\
$\begin{array}{l}\text { Limiares auditivos levemente alterados } \\
\text { Perda auditiva neurossensorial leve }\end{array}$ & $9(15 \%)$ & $4(10 \%)$ & 50 a 71 anos \\
\hline Total & $24(60 \%)$ & $11(27,5 \%)$ & 60 a 83 anos \\
\hline
\end{tabular}


Os dados obtidos na audiometria tonal foram classificados de acordo com Davis \& Silverman. ${ }^{10}$ Todos os sujeitos apresentaram resultados da imitanciometria compatíveis com os da audiometria tonal limiar.

Considerando os resultados da avaliação audiológica básica, foi possível agrupar os indivíduos avaliados em três categorias, que posteriormente tiveram os dados analisados, de forma independente, com a finalidade de determinar a qualidade auditiva dos mesmos. Desta forma, os sujeitos que tiveram resulta- dos audiológicos normais passaram a ser denominados neste estudo de grupo $\mathrm{A}(\mathrm{n}=10)$, os que tiveram resultados audiométricos levemente alterados, mas ainda considerados normais, segundo ISO 7029, ${ }^{11}$ grupo B $(\mathrm{n}=10)$, e aqueles com perda auditiva leve ou moderada de grupo $\mathrm{C}(\mathrm{n}=20)$.

A anamnese permitiu verificar dados importantes que caracterizam as queixas e condições de escuta dos sujeitos e é apresentada na tabela 2, já se considerando a subdivisão dos grupos citada anteriormente.

Tabela 2 - Número de sujeitos de acordo com queixas auditivas e de escuta. Tuiuti, PR, 2007.

\begin{tabular}{lccc}
\hline & $\begin{array}{c}\text { Grupo A } \\
(\mathrm{n}=10)\end{array}$ & $\begin{array}{l}\text { Grupo B } \\
(\mathrm{n}=10)\end{array}$ & $\begin{array}{c}\text { Grupo C } \\
(\mathrm{n}=20)\end{array}$ \\
\hline $\begin{array}{l}\text { Não refere dificuldade auditiva } \\
\begin{array}{l}\text { Refere dificuldades para compreender a } \\
\text { fala em ambientes confortáveis } \\
\text { acusticamente }\end{array}\end{array}$ & $0(0 \%)$ & $4(40 \%)$ & $16(80 \%)$ \\
$\begin{array}{l}\text { Refere dificuldades para compreender a } \\
\text { fala em ambientes ruidosos }\end{array}$ & $4(40 \%)$ & $4(40 \%)$ & $18(90 \%)$ \\
$\begin{array}{l}\text { Refere dificuldades para compreender a } \\
\text { fala na presença de 2 ou mais } \\
\text { interlocutores }\end{array}$ & $0(0 \%)$ & $5(50 \%)$ & $18(90 \%)$ \\
\hline
\end{tabular}

Com relação ao exame de processamento auditivo, observamos que em todos os grupos houve a identificação de algum tipo de alteração de percepção auditiva. Os resultados obtidos nos testes SSW e Dicótico de dígitos per- mitiram classificar o grau desta alteração de acordo com critérios de Pereira, ${ }^{12}$ o ouvido mais afetado e a condição de escuta, ou seja, habilidade auditiva mais alterada. Estes dados estão representados nos gráficos 1,2 3 . 


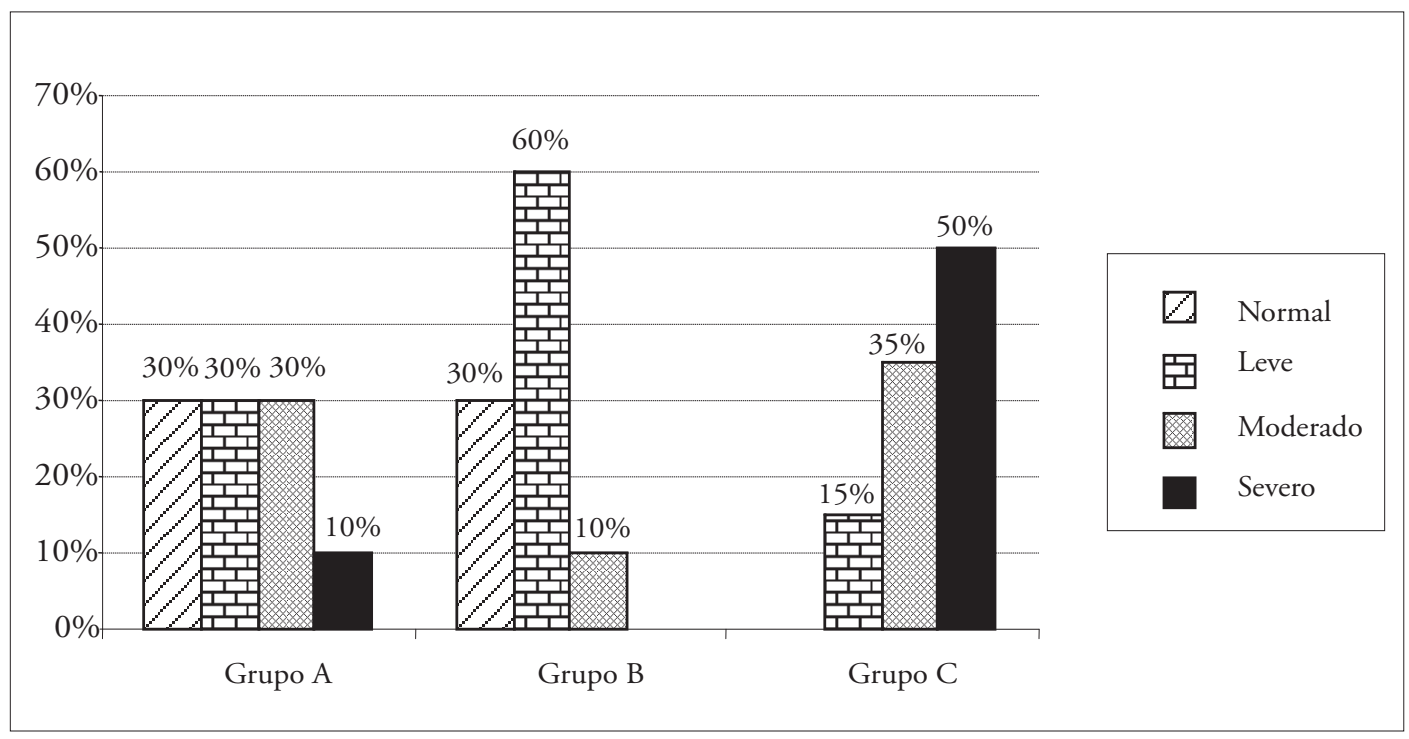

Gráfico 1 - Número de sujeitos de acordo com o grau da alteração do processamento auditivo. Tuiuti, PR, 2007.

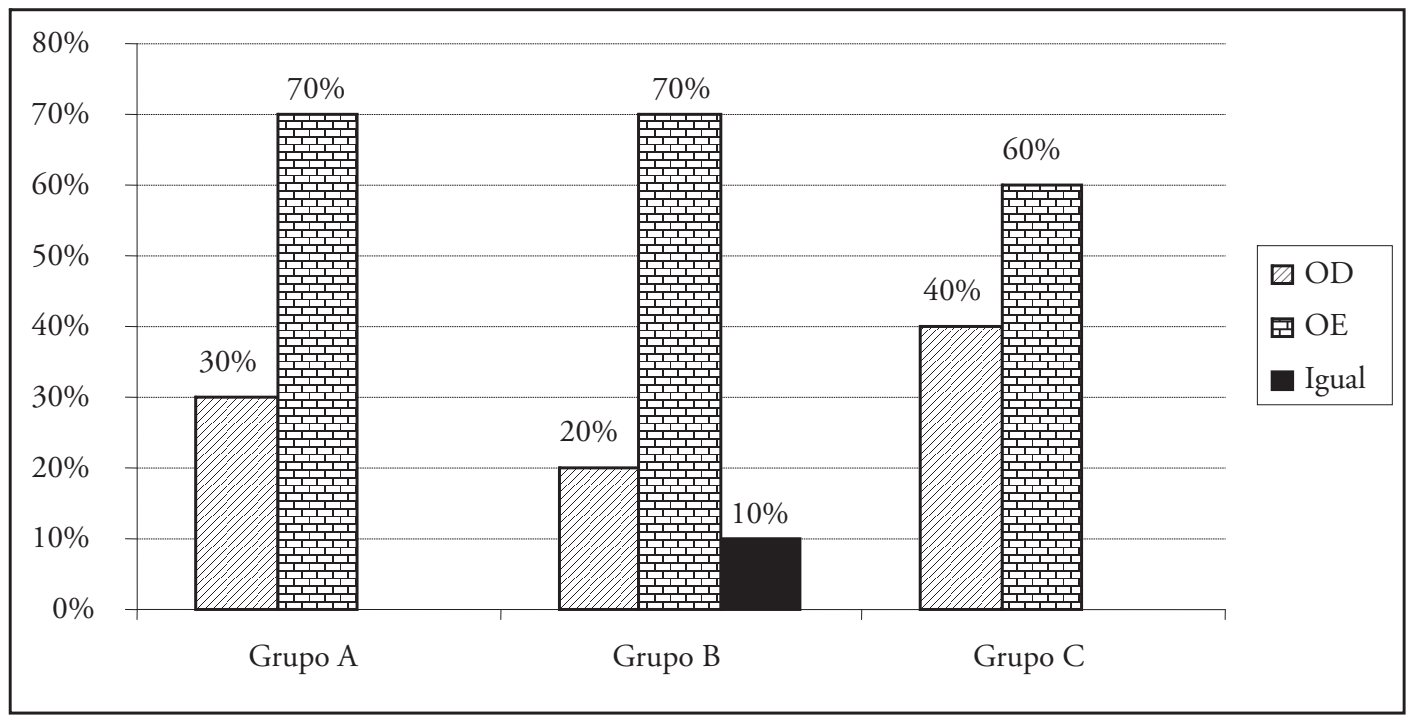

Gráfico 2 - Número de sujeitos de acordo com o ouvido mais alterado. Tuiuti, PR, 2007. 


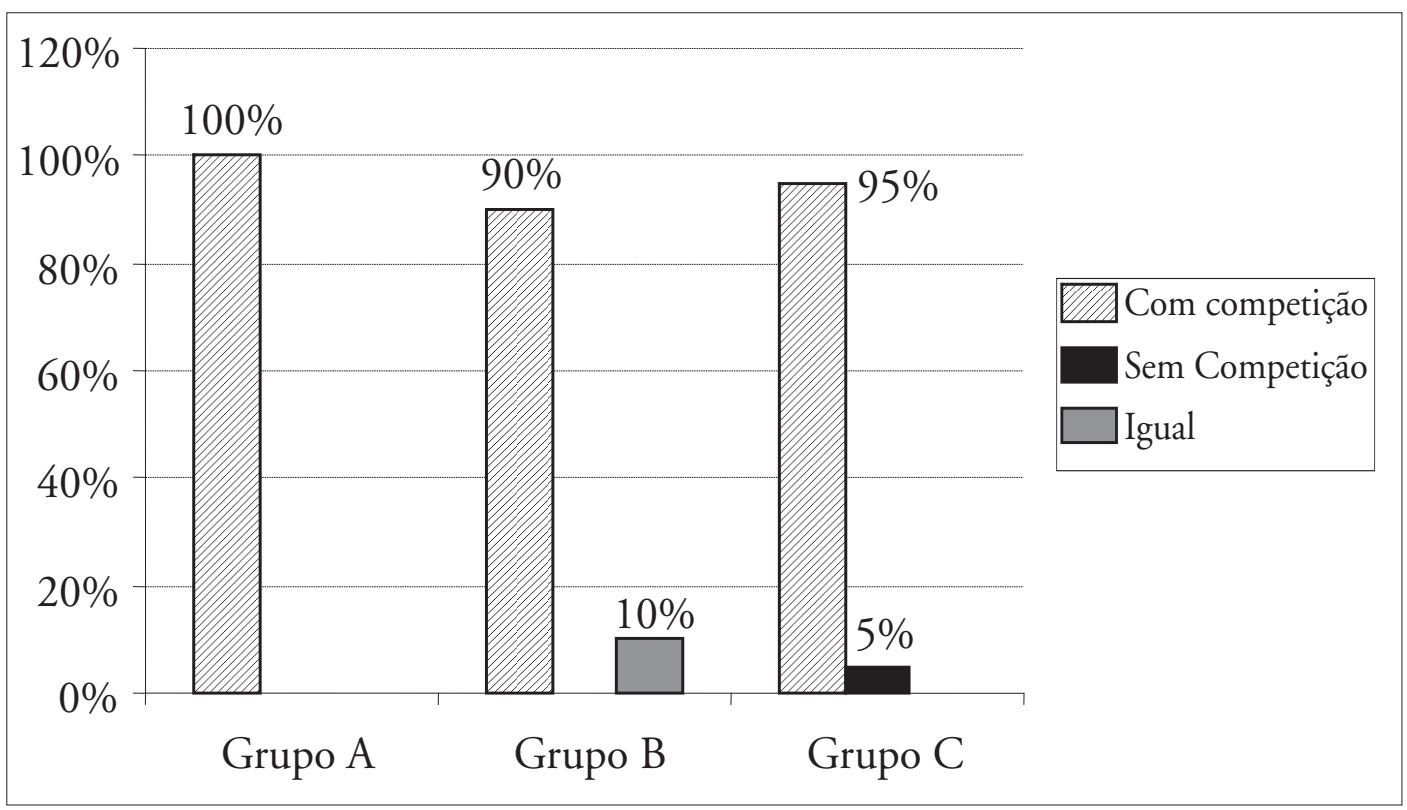

Gráfico 3 - Número de sujeitos e a dificuldade na condição de escuta. Tuiuti, PR, 2007.

Com relação ao ouvido mais afetado, registrou-se diferença estatisticamente significante $(\mathrm{p}=0,0005)$, quando comparados os dados do grupo $\mathrm{C}$, que diferem significativamente dos grupos $\mathrm{A}$ e B, ou seja, os sujeitos do grupo $\mathrm{C}$ tiveram pior desempenho nos exames de processamento auditivo quando o inputse deu pelo ouvido esquerdo.

\section{DISCUSSÃO}

Buscando atingir o objetivo proposto no início deste estudo, analisamos as respostas auditivas aos testes auditivos periféricos e centrais dos sujeitos avaliados, e observamos, como dado mais relevante, que independentemente da presença da perda auditiva peri- férica (vide tabela 1), as alterações de processamento para a escuta dicótica apareceram nos três grupos avaliados (vide gráfico 1), o que permite inferir que o envelhecimento das vias auditivas centrais está diretamente relacionado à dificuldades de escuta normalmente relatadas por populações idosas.

Por escuta dicótica entende-se a capacidade que o indivíduo possui de escutar sinais de fala, ou outros, pelos dois ouvidos ao mesmo tempo, em situações de fala degradada, ou seja, na presença de estímulos competitivos. Esta tarefa é realizada constantemente e sem dificuldades no dia a dia das pessoas, em situações como salas de aula, conversas em grupo, nas igrejas e nas próprias residências. Normalmente as pessoas conseguem 
focalizar a atenção no que é importante e relegam para segundo plano aquilo que não interessa no momento, apesar de estarem sempre atentas a todos os sons presentes no ambiente. Desta forma, o que registramos nesta pesquisa é que em todos os grupos houve dificuldades em escuta dicótica.

Outro aspecto relevante a ser pontuado foi o fato de que, com o avanço da idade, aumentam as queixas auditivas e aumentam também as dificuldades audiológicas, observadas tanto em nível auditivo periférico quanto central (vide tabela 1 e gráfico 1).

Todos os indivíduos do grupo A referiram ouvir bem, porém $70 \%$ deles apresentaram alteração de percepção no exame de processamento. $\mathrm{O}$ não-aparecimento dessa queixa auditiva se deve ao nível de audição normal encontrado nesses indivíduos, porém os resultados dos exames revelam que esta população já apresenta dificuldades de percepção, principalmente no que se refere ao escutar a fala em situação acústica degradada.

O grupo B, em sua maioria (80\%) acha que ouve bem, apesar de os indivíduos apresentarem rebaixamento auditivo quando considerados os padrões sugeridos por Davis \& Silvermann. ${ }^{10}$ Este dado se explica em função de a perda auditiva acometer as frequências altas, responsáveis mais pela inteligibilidade de fala do que pela sensação auditiva propriamente dita. Neste grupo também se registrou alteração de processamento auditivo em $70 \%$ dos sujeitos, mais especificamente na habilidade de atenção seletiva.
Já 85\% dos idosos do grupo C responderam que não escutam bem. Trata-se, portanto, da perda auditiva periférica interferindo no processo de comunicação. É no grupo C que aparecem os relatos significativos de dificuldades para compreender a fala, para escutar em ambientes ruidosos e de dificuldades para se comunicar quando há dois ou mais interlocutores (vide tabela 2 ), sendo que $100 \%$ dos sujeitos deste grupo falharam no exame de processamento auditivo. Estes resultados estão compatíveis com a literatura, de acordo com Freitas \& Oliveira, ${ }^{13}$ que afirmaram que é comum a prevalência da perda auditiva em aproximadamente $46 \%$ em indivíduos de 48 a 92 anos, tornando-se mais frequente a partir dos 55 anos de idade, atingindo cerca de $14 \%$ da população entre $60-64$ anos e $50 \%$ a $60 \%$ acima dos 80 anos.

Os testes dicóticos aplicados nesta pesquisa permitiram verificar se as pessoas avaliadas possuem algum problema de percepção auditiva. Em caso positivo, qual o grau desta alteração e qual a habilidade auditiva mais comprometida.

Em relação ao grau, Pereira ${ }^{14}$ refere que esta pode ser leve, moderada ou severa, e que quanto pior for a classificação, mais difícil é o processo de comunicação com o indivíduo afetado.

No que se refere às habilidades auditivas, fazem parte do processo de escuta as capacidades de realização de análise e síntese (ou fechamento auditivo), organização de eventos sonoros no tempo (ou memó- 
ria de curto prazo) e figura-fundo (ou atenção seletiva).

Conforme afirmamos anteriormente, em todos os grupos avaliados, foi detectado algum tipo de alteração do processamento auditivo para a escuta dicótica. Mesmo no grupo A, no qual os indivíduos não apresentam queixas em relação à percepção auditiva (tabela 2), observa-se alguma alteração de processamento, e como não há presença de perda auditiva, nem rebaixamento auditivo nas frequências altas, as alterações encontradas podem estar relacionadas ao envelhecimento do sistema auditivo.

No grupo $B$ há presença de rebaixamento auditivo nas frequências altas, podendo estes déficits de grau leve e moderado estar relacionados com a alteração da percepção auditiva, como também com o comprometimento periférico apenas nas altas frequências.

Os resultados obtidos nos grupos $\mathrm{A}$ e $\mathrm{B}$ vão de encontro à literatura pesquisada, tendo em vista que Bess, Hedley-Williams e Lichtenstein ${ }^{15}$ relatam que a dificuldade na compreensão de fala entre os idosos parece ser acentuada mesmo entre ouvintes normais quando a tarefa de escuta se torna mais difícil por meio da degradação do sinal de fala. Em se tratando da presença de rebaixamento auditivo nas altas frequências, a percepção da fala torna-se difícil, principalmente devido à concentração maior de consoantes nesta faixa de frequência.

No grupo C, todos os idosos apresentaram alteração do processamento auditivo, confirmando estudos que relataram que, no idoso, a função auditiva central pode estar reduzida por uma perda geral de neurônios em todo o sistema nervoso. ${ }^{16,17}$

Os variados graus de alteração encontrados na população do Grupo $\mathrm{C}$ podem estar relacionados com a presença de perda auditiva periférica. Segundo Katz, Tillery e Mec$\mathrm{ca},{ }^{18} \mathrm{a}$ associação entre fatores periféricos e centrais aumenta o desafio para o paciente e, desta forma, ele acaba cometendo mais erros. No entanto, os autores Jerger et al..$^{19}$ discordam desta afirmativa e relatam que a alteração do processamento auditivo parece ser independente da perda de sensibilidade periférica, mas segundo os mesmos, a diminuição na velocidade do processamento da informação, muito frequente na população idosa, pode afetar significativamente o desempenho da percepção da fala.

Assim como a diminuição auditiva, a dificuldade de entender o que as pessoas falam, mesmo em ambientes confortáveis acusticamente, é uma queixa muito comum entre os idosos e esta se deve à própria configuração audiométrica desses indivíduos. Nos grupos B e C, onde há alteração de limiares auditivos, percebe-se aumento de queixas relacionadas à compreensão da fala. Pedalini et al. ${ }^{20}$ afirmaram que essa queixa se deve à configuração audiométrica da perda auditiva, que, no caso da presbiacusia, atinge as frequências altas, tornando difícil a percepção dos fonemas consonantais. Entretanto, os mesmos autores asseguram que esta queixa nem sempre pode ser explicada exclusivamente pela perda auditiva, poden- 
do ser atribuída a alterações do sistema nervoso central. Quintero, Marotta e Marone ${ }^{21}$ afirmaram que as diversas mudanças que ocorrem nas vias auditivas periféricas e centrais do indivíduo idoso possivelmente interferem na sua habilidade de processar eficientemente a fala que recebe, determinando com frequência sua dificuldade de compreensão, independentemente do grau da perda auditiva.

Duas situações de escuta difícil foram bem identificadas nesta pesquisa: a primeira, relativa à existência de dificuldades de compreensão frente a vários interlocutores; a segunda, relativa à dificuldade de compreender a fala em ambientes degradados, onde há ruído de fundo importante.

Quando, num mesmo ambiente, vários interlocutores competem entre si, dificultase a tarefa de atenção seletiva ou figura-fundo auditiva. Nenhum sujeito do grupo A afirmou ter esta dificuldade, porém $50 \%$ de idosos do grupo $\mathrm{B}$ e $90 \%$ do grupo $\mathrm{C}$ reclamam que nestas condições é mais difícil comunicar-se. A situação de fala com mais de um interlocutor dá uma ideia de outros ruídos de fala, isto é, várias pessoas conversando ao mesmo tempo, e isso já representa certo ruído competitivo. Em se tratando de grupos com perda auditiva (grupo C), mesmo que restrita às altas frequências (grupo B), a situação de escuta deixa de ser ideal, pois agora o indivíduo terá outros sons de fala para prestar atenção e discriminar.

Quando o ambiente sonoro não é o ideal, no caso de haver ruído competitivo, como motores funcionando, eletrodomésticos ligados, barulho de carros e outros veículos, por exemplo, as dificuldades aumentaram. No grupo $\mathrm{A}, 10 \%$ afirmaram que não ouvem bem em ambientes ruidosos; no grupo $\mathrm{B}$, $40 \%$ dos idosos afirmaram sentir dificuldade; e no grupo C, foram $80 \%$. Segundo Northern \& Downs, ${ }^{22}$ ruídos de fundo comuns variam de aproximadamente 35 a 68 $\mathrm{dB}$ NPS, e para adultos com audição normal, a situação na qual o ruído é $10-15 \mathrm{~dB}$ abaixo do nível da fala não cria dificuldade de escuta, pois o ouvinte pode preenchê-lo com as pistas acústicas que faltam. Entretanto, em se tratando de idosos, a discriminação da fala torna-se difícil, principalmente, devido à perda auditiva mais restrita às altas frequências, onde se concentram o maior número de fonemas consonantais.

Soncini, Costa e Oliveira ${ }^{23}$ explicaram que adultos com mais de 50 anos, normoouvintes, necessitam de maior intensidade na apresentação das sentenças no silêncio e no ruído para alcançar o mesmo percentual de reconhecimento de fala que os adultos jovens também normo-ouvintes. Desta forma, acreditam que o processo de envelhecimento é um fator que interfere no reconhecimento da fala no silêncio e no ruído, quando a audição periférica é normal. Zamperlini, Kyrillos e Santos ${ }^{24}$ também afirmam que a diminuição na inteligibilidade da fala, sobretudo em ambientes ruidosos, é decorrente das alterações centrais que em geral se iniciam aos 60 anos.

Quando analisamos os dados relativos ao ouvido mais afetado (vide gráfico 2), ve- 
rificamos que houve diferença estatisticamente significante quando se compararam os dados dos grupos A e B com os do grupo C. A literatura pesquisada por Hallgren et al. ${ }^{25}$ refere que estudos realizados com testes dicóticos em idosos encontraram problemas quando o inputse dava pelo ouvido esquerdo. Os referidos dados também concordam com os autores, ${ }^{26}$ que estudaram casos de adultos destros e encontraram vantagem auditiva do ouvido direito para processar estímulos dicóticos verbais, sugerindo déficit na transferência da informação via corpo caloso. Em outro caso estudado pelas mesmas autoras, evidenciou-se diferença de desempenho entre os dois ouvidos no teste dicótico, com vantagem auditiva do ouvido direito de $12 \%$, podendo este dado ser explicado pelo início do processo de envelhecimento das vias auditivas centrais.

Os dados coletados a respeito do ouvido mais alterado são essenciais para se fazer o topodiagnóstico da alteração, além de auxiliar a escolha do ouvido a ser protetizado em casos de adaptação unilateral, onde, de acordo com a literatura pesquisada, ${ }^{9}$ a orelha com menor comprometimento da função auditiva central seria a mais indicada para a adaptação.

Para finalizar a análise dos dados, é relevante demonstrar que praticamente todos os sujeitos da amostra (90\%) apresentaram dificuldade quando a condição de escuta foi competitiva (vide gráfico 3). Desta forma, quando os sujeitos desta pesquisa foram submetidos à fala em situação degradada, isto é, em situações de escuta não ideal, os mesmos apresentam dificuldade para compreendê-la.

Conclui-se, portanto, que a idade, além da perda auditiva, contribui para o baixo desempenho de idosos na percepção de fala na presença de ruído, em que tarefas auditivas mais complexas que envolvem estímulos de fala apresentados em condições menos favoráveis demonstram deterioração maior nos resultados de indivíduos idosos do que em indivíduos mais jovens. Desta forma, o processo de envelhecimento é um fator que interfere no reconhecimento $d a$ fala em situações degradadas, mesmo quando a audição periférica é normal.

\section{CONCLUSÃO}

A pesquisa em questão revelou, através de seus resultados, a existência de uma relação importante entre o processamento auditivo com o fator envelhecimento, tendo em vista que:

- $85 \%$ da amostra apresentaram alguma alteração de processamento auditivo, independentemente da presença de perda auditiva periférica.

- A queixa auditiva relativa à dificuldade de ouvir em locais não ideais de escuta é preponderante na amostra estudada, o que evidencia dificuldades com a habilidade de atenção seletiva na população idosa.

- Na medida em que aumenta a faixa etária da amostra, aumentam as dificuldades 
auditivas e o grau de alteração do processamento auditivo.

- Na medida em que aumenta a perda auditiva, aumenta o grau de alteração do processamento auditivo.

- No que concerneà condição de escuta competitiva e não-competitiva, a maioria dos indivíduos, em todos os grupos, apresentou resultados piores, estatisticamente significantes, em condição de escuta competitiva. A alteração do processamento auditivo aparece com maior significância quando o imput se dá pelo ouvido esquerdo.

\section{REFERÊNCIAS}

1. Carvalho JAM, Andrade FCD.

Envejecimiento de la población brasileña: oportunidades y desafíos. Anais do Encuentro Latinoamericano y Caribeño sobre las Personas de Edad. Santiago: CELEDADE; 1999.p. 81-102.

2. Nakatani. Perfil sócio-demográfico e avaliação de idosos atendidos por uma equipe de saúde da família na periferia de Goiânia-GO. Revista Sociedade Brasileira de Clinica Medica 2003; 1(5): 131-6.

3. Papaléo Netto M. Gerontologia: a velhice e o envelhecimento em visão globalizada. São Paulo: Atheneu; 2002.

4. Costa SS, Cruz OL, Oliveira JA. Otorrinolaringologia. Porto Alegre: Artes Médicas; 1997. p. 51.

5. Corso JF. Presbyacusis, hearing aids and aging. Audiology 1977; 16 (2): 146-63.

6. Parra VM, Iório MCM, Mizahi MM, Baraldi GS. Testes de padrão de frequência e de duração em idosos com
Tendo em vista os resultados encontrados neste estudo, pode-se inferir a importância da avaliação audiológica completa, que envolva testes periféricos e centrais, em populações idosas, com vistas a uma reabilitação auditiva adequada, especialmente direcionada para as habilidades auditivas prejudicadas. Os resultados audiológicos podem auxiliar no processo de seleção e adaptação de próteses auditivas e, assim, mais benefícios podem ser apresentados a esta população, minimizando os efeitos do envelhecimento sobre a percepção auditiva. sensibilidade auditiva normal. Rev Bras Otorrinolaringol 2004 jul/ago; 70 (12): 517-23.

7. Katz J. The use of staggered spondaic words for assessing the integrity of central auditory nervous system. Journal Audiology Research 1962; 2: 327-37.

8. Batista FP, et al. Análise comparativa do Índice de Reconhecimento de Fala em pacientes presbiacúsicos com e sem recrutamento. Revista CEFAC : atualização científica em fonoaudiologia 2006; 8 (2): 230-4.

9. Helfer K. Measuring speech recognition in older adults. In: Mendell LL, Danhauer JL. Audiologic evaluation and management and speech perception assessment. San Diego, CA: Singular; 1997.p.133-48.

10. Davis H, Silvermann RS. Hearing and deafness. New York: Holt, Rinehart e Wiston; 1970. 
11. Fortier $\mathrm{P}$, et al. Mise à jour du système standardize d'analyse des resultants des examens auditifs realizes auprés des travaileurs exposés au bruit. Guide d'accompagnement (version préliminaire 1.1).Québec: Centre d'expertise en dépistage. Institut national de santé publique du Québec; 2000. 1-87 p.

12. Pereira, LD. Identificação de desordem do processamento auditivo central através de observação comportamental: organização de procedimentos padronizados. In: Schochat E. Processamento auditivo. São Paulo: Lovise; 1996. p. 43-56.

13. Freitas MR, Oliveira JAA. Audiometria de respostas evocadas de tronco cerebral em indivíduos idosos com e sem presbiacusia. Rev Bras Otorrinolaringol 2001 mar/abr; 67 (5): 171-8.

14. Pereira LD. Processamento auditivo central: abordagem passo a passo. In: Pereira LD, Schochat E. Processamento auditivo central: manual de avaliação. São Paulo: Lovise; 1997 . p. 49-59.

15. Bess FH, Hedley-Williams A, Lichtenstein MJ. Avaliação audiológica em idosos. In: Musiek FE, Rintelmann MJ. Perspectivas atuais em avaliação auditiva. São Paulo: Manole; 2001. p. 343-69.

16. Baran JA, Musiek FE. Avaliação comportamental do sistema nervoso auditivo central. In: Musiek FE, Rintelmann WF. Perspectivas atuais em avaliação auditiva. São Paulo: Manole; 2001. p. 371-409.

17. Cooper JC, Gates GA. Hearing in the Elderly-The Framingham Cohort, 983-
1985: Part II. Prevalence of Central Auditory Processing Disorders. Behavioral Audiology. Ear and Hearing 1991 oct; 12 (5): 304-11.

18. Katz J, Tillery KL, Mecca F. Uma introdução ao processamento auditivo. In: Lichtig I, Carvalho RMM. Audição: abordagens atuais. São Paulo: Pró-Fono; 1997.p. 146-72.

19. Jerger J, et al. Speech understanding in the elderly. Ear and Hearing 1989; 79-89.

20. Pedalini MEB, et al. Análise do perfil audiológico de idosos através de testes de função auditiva periférica e central. Rev Bras Otorrinolaringol 1997 set/out; 63 (9): 489-96.

21. Quintero SM, Marotta MRB, Marone SAM. Avaliação do processamento auditivo de indivíduos idosos com e sem presbiacusia por meio do teste de reconhecimento de dissílabos em tarefa dicótica - SSW. Rev Bras Otorrinolaringol $2002 \mathrm{jan} / \mathrm{fev}$; 68 (5): 28-33.

22. Northern JL, Downs MP. Audição na infância. Rio de Janeiro: Guanabara; 2005. 3-27 p.

23. Soncini F, Costa MJ, Oliveira TMT. Influência do processo de envelhecimento no reconhecimento da fala em indivíduos normo-ouvintes. Pro-Fono 2003 set/dez; $15(3): 34-8$.

24. Zamperlini HBL, Kyrillos LCR, Santos MCF. A comunicação na terceira idade: características e reflexões. In: Lagrotta MGM, César CPHAR. A Fonoaudiologia nas instituições. São Paulo: Lovise; 1997. 41-8 p.

25. Hallgren M, et al. Cognitive effects in dichotic speech testing in elderly persons. Ear and Hearing $2001 \mathrm{abr} ; 22$ (2): 120-9.

26. Sanchez ML, Alvarez AMM, Cabete CF, Frazza MM. Avaliação do processamento auditivo em adultos. Acta AWHO 2003 jan/mar; 21: 41-8. 